NASA Technical Memorandum 86176

\title{
Consequences of Hot Gas in the Broad Line Region of Active Galactic Nuclei
}

T. Kallman and R. Mushotzky

MARCH 1985

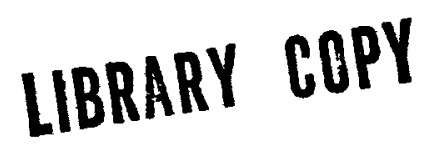

$\therefore \quad 1963$

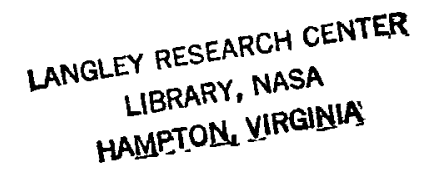


NASA Technical Memorandum 86176

\section{Consequences of Hot Gas in the Broad Line Region of Active Galactic Nuclei}

T. Kallman and R. Mushotzky

Goddard Space Flight Center

Greenbelt, Maryland 
We discuss models for hot gas in the broad line region of active galactic nuclei. The results of the two phase equilibrium models for confinement of broad line clouds by Compton heated gas as described by Krolik, McKee and Tarter (1982) are used to show that high Tuminosity quasars are expected to show Fe XXVI La line absorption which will be observable with spectrometers such as those planned for the future $X$-ray spectroscopy experiments. Two phase equilibrium models also predict that the gas in the broad 1 ine clouds and the confining medium may be Compton thick. We show that the combined effects of Comptonization and photoabsorption can suppress both the broad emission lines and X-rays in the Einstein and HEAO-1 energy bands. The observed properties of such Compton thick active galaxies are expected to be similar to those of Seyfert 2 nuclei. The implications for polarization and variability are also discussed.

Subject Headings: galaxies: nuclei -- quasars --radiation mechanisms 
I. INTRODUCTION

Several recent theoretical and observational developments suggest that hot $\left(T>10^{6} K\right)$ gas may be important in the broad emission line reqion of quasars and active galactic nuclei (AGN). Theoretical arguments concerning the thermal instability of photoionized gases (McCray 1979: Krolik, McKee and Tarter, 1982), together with the need for pressure confinement of broad line clouds (e.g. Mathews 1974) predict that the clouds will be bathed in a hot confining medium. If this is so, then the hot intercloud qas (ICM) may produce observable $X$-ray absorption and emission lines from ions such as hydrogen-like iron, in addition to continuum absorption edges.

Hot gas may al so have broad implications in determining the distinguishing features of the various different classes of AGN. If the qas is present in slightly larger quantities than predicted by thermal instability (two phase) models then Comptonization can suppress the highest energy continuum from the central source. Such Comptonized spectra will not have an unstable effect on gas in the broad line region (the broad emission line clouds may not form), and the X-ray continuum may also be photoabsorbed in the Comptonizing region. Such Compton thick objects may resemble Seyfert 2 's in their continuum properties.

In this paper we consider the spectral consequences of hot gas in the broad emission line region of AGN. We do this both in the context of the two phase models proposed for objects which show broad lines, and in the more general case when the continuum source is embedded in Compton thick material. In doing so, we employ standard "nebular" approximations for the transfer of continuum photons when the gas is Compton thin (see, e.g. Kallman and McCray 1982; Kallman 1984). Gas flows and dynamical models for broad line regions are considered only in so far as they affect the gas distribution in 
the broad line region. Narrow emission lines and radio frequency properties of AGN are not considered. Compton thin models are presented in $\$ I I$. The Compton thick case is considered in \$III. \$IV provides a discussion and summary of our results.

\section{SPECTRAL FORMATION IN THE QUASAR ICM}

\section{a) The Two Phase Model}

In this section we explore the potentially observable properties of the ICM in objects which are observed to have broad optical and UV emission 1 ines, i.e. quasars and Seyfert 1 galaxies. In such objects the correspondence between the ICM and the properties of the broad line emitting clouds has been discussed in detail by Krolik, McKee, and Tarter (1982; hereafter KMT). We present here a summary and confirmation of their results as a background for our later discussion.

The connection between the broad emission line clouds and the ICM is due to the fact that both are heated and photoionized primarily by continuum photons from a compact source at the center of the broad line region. The gas is assumed to exist in thermal equlibrium at constant pressure, $P$. The gas temperature and degree of jonization depend only on the shape of ionizina continuum spectrum and on a parameter proportional to the ratio of continuum radiation pressure to gas pressure, the ionization parameter $\Xi$. In terms of the incident flux, $F\left(\operatorname{erg~sec} \mathrm{sm}^{-1} \mathrm{~cm}^{-2}\right)$, the ionization parameter is defined as $\Xi=F / C P$. At large values of this parameter the gas is fully ionized and the temperature is determined by Compton and inverse-Compton scattering. The temperature in this case, denoted $T_{I C}$, depends on the shpae of the continuum spectrum and on the high and low energy boundaries of the spectrum. For 
example, if the spectral shape is a power law with (energy) index less than one $T_{I C}$ is sensitive to the high energy spectral cutoff. If the spectral index is greater than one, $T_{I C}$ depends on the cutoff at 1 ow energies. At small $\Xi$ the gas is recombined, and the temperature is approximately $10^{4} \mathrm{~K}$, owing to the strong cooling by atomic transitions of hydrogen, HeII, and C IV ions. At intermediate $\Xi$ the thermal equilibrium condition may allow both Comptonized and $10^{4} \mathrm{~K}$ temperatures to occur at the same value of $\Xi$. In this two phase region of parameter space the net cooling function of the gas satisfies Field's (1965) criterion for thermal instability. The thermally unstable zone is bounded by the critical ionization parameters, denoted $\Xi_{C}^{*}$ and $\Xi_{h}^{*}\left(\Xi_{C}^{*}>\Xi_{h}^{*}\right)$. The value of $\Xi_{c}^{*}$ is fixed by the atomic cooling properties of the gas, $\Xi_{C}^{*} \approx 10$. The value of $\Xi_{h}^{*}$ depends on $T_{I C}: \Xi_{h}^{*} \alpha T_{I C}{ }^{-3 / 2}$. The two phase zone is evanescent (i.e. $\Xi_{c}^{*} \leqslant \Xi_{h}^{*}$ ) for $T_{I C} \leqslant 10^{7} K$.

The two phase models are successful in accounting for the fact that quasar broad emission line clouds are observed to exist over a narrow range of ionization parameter. Comparison of observed broad 1 ine ratios with clour photoionization models reveals that clouds exist in the range of ionization parameters $0.3<\Xi<2$ (Kwan and Krolik 1981) for an ensemble of AGN with Iuminosities in the range $10^{44} \mathrm{erg} \mathrm{s}^{-1}-10^{47} \mathrm{erg} \mathrm{s}$. This range is rouqhiy congruent with the range of ionization parameters for which clouds and ICM can coexist via the two-phase models. It is well known from measurements of the line fluxes and sizes of the BLR that the filling factor of the dense clouds is $<<1$. Therefore there is not enough gas to fill the BLR at $10^{4} \mathrm{~K}$. . The rates of cloud evaporation and free expansion are sufficient to produce the ICM even if the clouds were somehow initially produced in the absence of such hot gas. 
The simple two phase model is subject to modification by a number of other processes which may be occuring in AGN. Sources of heating other than photoionization and Compton scattering, such as suprathermal particles or radio frequency heating, will increase the ICM temperature above $T_{I C}$ and extend the range of thermally unstable ionization parameters. Advection of material into the broad line region by gas flowing with a timescale comparable to the cooling and heating timescale can act either to enhance the instability (if the advected material is hot) or to partially suppress it (if the advected material is cooll). Evaporation of the clouds will tend to raise the cloud pressure over that of the ICM. Radiation pressure due to trapped line photons can disrupt clouds preferentially at large ionization parameters, thus effectively reducing $\Xi_{C}^{\star}$ (see also Weymann 1976). Al though all these processes are potentially important in determining the ICM properties, a detailed accounting of them depends sensitively on many poorly known properties of the broad line clouds (gas flow, spectrum of cloud sizes, relativistic particle flux, etc.). In what follows we adopt the point of view that the simple two phase model provides an approximate average description of the ICM, and we use this for purposes of quantitative estimates of the ICM properties. We also mention the potential qualitative modification to our results that will arise from the processes listed in this paragraph.

\section{b) Two Phase Models}

The existence of observable spectral features formed in the ICM depends on the degree of ionization of the ICM, and on the gas column density. The ion abundances depend on the gas temperature, $T \approx T_{I C} \approx 10^{7}-10^{8} \mathrm{~K}$, and on the shape of the ionizing continuum spectrum. The column density depends on the values of the critical ionization parameters, $\Xi_{c}^{*}$ and $\Xi_{h}^{\star}$, which also depend on 
the continuum shape, and on the distribution of gas pressure as a function of position. For a variety of plausible choices for continuum shape and pressure distributions the two phase model predicts the existence of features which will be observable with future X-ray spectroscopy experiments.

The value of the ICM temperature, TIC, depends on the detailed shape of the continuum from the central source. Observed AGN spectra typically have a power law spectral index $\alpha \approx 0.7$ (in eneray) between $1 \mathrm{keV}$ and several hundred keV (Mushotzky et al. 1980; Worrall et al. 1981: Primini et al. 1980; Rothschild et al. 1982) and $\alpha \approx 1.2$ between wavelength of $10 \mu$ and $1000 \AA$ (Malkan and Sargent 1980; Glass 1982, Glass et al. 1982). The spectra between $0.1 \mathrm{eV}$ and $500 \mathrm{keV}$ can be adequately represented by

$$
f_{\varepsilon}=A \varepsilon^{-1.2} e^{-\varepsilon / \varepsilon_{0}}+B \varepsilon^{-0.7}
$$

where $\varepsilon$ is the photon energy in $\mathrm{eV}$ and $\varepsilon_{0}=200 \mathrm{eV}$. The spectral softness parameter $A / B$ has a value in the range from $0.1(\mathrm{eV})^{1 / 2}$ to $1000(\mathrm{eV})^{1 / 2}$ (Zamorani et al. 1981; Reichert et al. 1982). This ratio is related to the conventionally used optical to $X$-ray spectral index, $\alpha_{0 x}$, according to $\alpha_{o x}=\frac{1}{2.605} \log A / B+0.698$. For $A / B=0.1(e V)^{1 / 2}, \alpha_{0 x} \approx 0.3$ : for $A / B=$ $1000(\mathrm{eV})^{1 / 2}, \alpha_{0 x} \approx 1.8$.

Figure 1 shows the range of $\Xi_{c}^{*}, \Xi_{h}^{*}$, and $T$ IC as a function of the spectral softness parameter $A / B$. These results were calculated using the photoionization program described by Kallman and McCray (1982) and Kallman (1984) using the element abundances of Kwan and Krolik (1982). The incident spectrum is assumed to extend from $0.1 \mathrm{eV}$ to $511 \mathrm{KeV}$. Compton heating and cooling are treated using the fully relativistic cross section and electron velocity distribution function. The range of broad line cloud ionization 
parameters inferred from the ensemble of observed quasar 1 ine ratios are shown as the dashed lines in the lower panel of this figure (Kwan and Krolik 1982). For $A / B \approx 0.1(\mathrm{eV})^{1 / 2}$ our model results are similar to those of the standard model of KMT; for $A / B=10(\mathrm{eV})^{1 / 2}$, corresponding to $\alpha_{\text {ox }} \approx 1$, our spectrum has roughly the same optical to X-ray flux ratio as the KMT standard spectrum; for this choice of spectrum we find $\Xi_{h}^{*}$ about a factor of 31 arger than KMT's. We attribute this difference to the fact that our spectrum is steeper in the hard $X$-ray spectral region than theirs.

The observed ensemble of radio loud quasars has a mean optical to $X$-ray flux ratio corresponding to $\alpha_{0 x} \approx 1.2$ or $A / B \approx 20(\mathrm{eV}) 1 / 2$. Radio quiet quasars have $\alpha_{O X} \approx 1.5$ or $A / B \approx 110(\mathrm{eV})^{1 / 2}$ (Zamorani et al. 1982). Fiqure 1 shows that in either case the range $\Xi_{h}^{*}<\Xi<\Xi_{C}^{*}$ misses the inferred observed cloud ionization parameters entirely, although the width of the allowed ionization parameter region, $\log \left(\Xi_{c}^{*} / \Xi_{h}^{*}\right)$, is comparable to the observationally inferred width. The predicted ionization parameter range can be brought into accord with the observed one if the effects of cloud evaporation are considered. Evaporation tends to increase the cloud pressure relative to the ICM by a factor of $\sim$ a few (KMT), thus shifting the clouds to lower ionization parameter. A shift of a factor of 6 in cloud pressure and hence ionization parameter, is sufficient to bring the two phase models into aareement with the cloud observations. Figure 1 also shows that for $A / B \geq 300(\mathrm{eV})^{1 / 2}$, corresponding to $\alpha_{0 x} \geq 1.65$, the two phase region is evanescent. This is consistent with the observed paucity of objects with spectra in this range: the only known measurement of $\alpha_{o x}>1.9$ may be a consequence of $x$-ray absorption by intervening matter (Elvis and Fabbiano, 1984).

Since the value of $\Xi_{h}$ is dependent on the spectrum at very high $1 \sim 0.5$ $\mathrm{MeV}$ ) and very low (1-10 m) photon energies, and since photons in these enerqy 
ranges do not contribute directly to the cloud ionization and broad line emission, the correspondence between the inferred observed cloud ionization parameters and the computed $\Xi^{*}$ provide constraints on the true AGN spectrum at very high and very low energies. Thus, our calculations rule out the existence of a steep (index >1.1) IR continuum extending to wavelengths greater than $\sim 10 \mu$ in broad line objects and requires broad line objects to be hard X-ray sources.

Al though the two phase models predict the run of ICM temperature and ionization as a function of $\Xi$, the distribution of gas pressures as a function of position depends on a detailed dynamical model for the gas flows in AGN. Various suggestions for the net flow in the broad line cloud region include spherical accretion (e.g. Krolik and London 1982) and radiatively driven outflow (Mathews 1982, Beltrametti 1981 and references therein). In either case the gas pressure may be represented roughly as a power 1 aw function of distance from the central continuum source, $P \alpha R^{\beta}$. Free fall predicts $\beta=$ $-3 / 2$, constant velocity outflow predicts $\beta=-2$, and radiatively driven outflow requires $\beta<-2$ (Mathews 1982). The relative merits of these models are influenced by the fact that the range of pressures which can explain the properties of observed broad emission lines do not vary by more than an order of magnitude over the ensemble of AGN. The range of pressures occurring within any given object may be much less. If we adopt the point of view that the broad line pressure can vary at most by a factor of 10 across the range of parameter space $\Xi_{c}^{*}>\Xi>\Xi_{h}^{*}$, then $B$ is determined by the relation

$$
\beta=\frac{-2}{1+\frac{\Delta \log \Xi}{\Delta \log P}} .
$$


If $\Delta \log \Xi=\log \Xi_{c}^{*}-\log \underset{h}{\Xi_{h}^{*}}$ and if $\Xi_{c}^{*}=2$ and $\Xi_{h}^{*}=0.3$ (Kwan and Krolik 1982), then $\beta$ must 1 ie in the range $-1 \leqslant \beta \leqslant 10$. It is interesting to note that the two phase models further constrain the pressure distribution via the mean density of the clouds plus ICM. That is, if the mean density is such that the gas cannot all exist at $10^{4} K$ throughout the region of thermal instability as has been shown by KMT, then the region of ionization parameter space $\Xi<\Xi^{*}$ must be forbidden. Thus the pressure distribution in the broad line region must be such that the pressure cannot be high enough for the ionization parameter to be less than $\Xi_{h}^{*}$. For a power law distribution of pressure with radius throughout the broad line region the power law index must be $\beta \approx-2$ in order to satisfy this constraint. Finally, we note that even though many of these arguments concerning the broad 7 ine region density distribution apply to the $10^{4} \mathrm{~K}$ clouds rather than to the ICM, the viscous coupling between clouds and ICM is sufficiently strong that velocity differences between the two components of more than $\sim 100 \mathrm{~km} \mathrm{sec}^{-1}$ are not expected. Thus these dynamical arguments apply equally to clouds and ICM, although hot gas may exist in regions devoid of clouds.

Given the conflicting arguments and constraints regarding the broad line region pressure distribution, we attempt to explore a variety of plausihle distributions which illustrate the range of ICM properties expected. In all cases we assume that the region of interest is bounded by the critical ionization parameters required by the two phase models, $\Xi_{c}^{*}$ and $\Xi_{\hbar}^{\star}$. Thus our estimates do not rely on the presence of hot gas in regions other than where it is required by the existence of clouds.

The column density of a given ion through a spherical shell bounded by $\Xi_{c}^{\star}$ and 涝 is 


$$
N=\frac{1}{2+\beta}\left(\frac{L P}{4 \pi c \Xi_{h}^{\star}}\right)^{1 / 2} \frac{1}{k} \int_{1}^{\Xi^{\star} / \Xi_{h}^{\star}} \frac{d x g(x)}{T(x)} x^{\frac{2 \beta+3}{\beta+2}}
$$

for a power law pressure distribution, where $P$ is the pressure at $\Xi=\Xi_{h}, L$ is the source luminosity, and $T$ is the gas temperature and $g$ is the fractional ion abundance relative to hydrogen. The ion column densities are weighted toward ionization parameters $\Xi \simeq \Xi_{\hbar}$ if $\beta>-2$. Observed quasar line ratios indicate that clouds exist primarly near $\Xi \geq \Xi_{\mathrm{h}}^{*}$ (Kwan and Krolik, 1982: KMT), suggesting that $\beta \leqslant-2$ does not occur. This suggests that strongly accelerated winds are less likely than constant velocity (or deceleratina) winds or virialized flow of the ICM. Since the ionization and temperature across the two phase region vary by a factor of only a few, the ion column densities for $\beta<-2$ are similar to those for $\beta>-2$. If $\beta \cong-2$, as in a constant velocity wind, is the true ICM pressure distribution then some other physical effect, such as a cutoff at large distance from the continuum source due to a scarcity of gas, must determine the ion column densities. Perhaps the simplest pressure distribution is $B=0$, so that $P=$ constant. In this case, since $\Xi_{C}^{*} \gg \Xi_{h}^{*}$, the column density is:

$$
N \approx 1.5 \times 10^{24} \mathrm{~cm}^{-2} \sqrt{\frac{L_{46^{P}} 14}{n_{h}^{x}} \frac{1}{8}}
$$

where $L_{46}$ is the luminosity in units of $10^{46} \mathrm{erg} \mathrm{sec}^{-1}, \mathrm{P}_{14}$ is ICM pressure in units of $10^{14} \mathrm{~cm}^{-3} \mathrm{~K}$, and $T_{8}$ is the gas temperature in units of $10^{8} \mathrm{~K}$.

Equation (4) provides an estimate of $N$ for $B$ not too different from zero. For the remainder of this section we will concentrate first on $\beta=0$ models, and then argue that their results will be substantially unchanged for $B$ in the range $|\beta|<2$. Finally, we discuss the effects of pressure distributions which do not fall within this range. 


\section{c) Spectral Results}

For our choice of incident spectrum the ICM is typically highly ionized, and the abundance of trace ions such as the hydrogen-like species of the trace element $0, S i, S$, and Fe depend sensitively on both the shape of the spectrum and on the range of ionization parameters in the ICM. The Fe XXVI and 0 VIII fractional abundances have maximum values, near $\Xi=\Xi_{h}^{*}$, of $\sim 10^{-1}$ for all cases except for the softest spectra, $A / B=1000(\mathrm{eV})^{1 / 2}$, for which the $\mathrm{Fe}$ XXVI and 0 VIII abundance can reach 0.4. The total column density of Fe XXVI is displayed in Figure 2, as a function of the spectral softness A/B through a constant pressure region bounded by $\Xi_{h}^{*}$ at large radius and $\Xi_{c}^{*}$ at small radius. Also shown are the column densities of 0 VIII and the total nucleon column density. From this figure it is apparent that the constraint that the ICM be Compton thin implies that $\left(L_{46} P_{14}\right)^{1 / 2}(A / B)^{1 / 4}<1.5$.

The cross section for absorption of photons at the center of the $L \alpha$ analog resonance line of a hydrogenic ion is (e.g. Bethe and Salpeter 1957)

$$
\sigma_{\text {ine }}=\frac{\pi e^{2}}{m_{e} c} \frac{f h}{\Delta \varepsilon}
$$

where $e, h, m_{e}$, and $c$ are the familiar constants, the oscillator strength in the non relativisitic approximation is $f=0.4162$, and $\Delta \varepsilon$ is the line width. If $\Delta \varepsilon$ is measured in eV then the cross section is $\sigma_{1 i n e}=4.6 \times 10^{-17} \mathrm{~cm}^{2} / \Delta \varepsilon e V$. Thus an Fe XXVI La absorption line broadened by gas motions of $10,000 \mathrm{Km} \mathrm{sec}{ }^{-}$ 1 , as suggested by the UV line widths, requires an ion column density of $5 \times$ $10^{+18} \mathrm{~cm}^{-2}$ in order to be optically thick. The ions 0 VIII, Si XIV, and $S$ XVI require column densities of $4.8 \times 10^{17} \mathrm{~cm}^{2}, 1.5 \times 10^{18} \mathrm{~cm}^{2}$, and $1.9 \times 10^{18}$ $\mathrm{cm}^{2}$, respectively, in order to be optically thick with this choice of 
broadening. The optical depths required for thermalization of resonance line photons are much greater than those obtainable in the ICM. Therefore, if the gas motions are turbulent or random, absorption by material along the line of sight to the source will be filled in by scattered emission (the net effect of the loss of scattered photons by absorption in the central source is likely to be negligible). If the gas has a net velocity toward or away from the central source an observable P-Cygni, or inverse P-Cygni, line profile is expected (this possibility has also been suggested by Shields and McKee, 1971). The absorption equivalent width of such a line will be $W_{a}=\sqrt{\pi} \tau_{a} \Delta \varepsilon$ for $\tau_{a}<1$ (Basko et a1. 1980). If $\tau_{a}=0.1$ at Fe XXVI La we expect $W_{a}=40 \mathrm{eV}$ with our previous choice of broadening. Features with this strength will be observable with future $X$-ray spectrometers. Figure 2 shows that for Fe XXVI $L_{\alpha} \tau_{a}=0.1$ is exceeded for all plausible choices of illuminating spectrum if $L_{46} P_{14}=1$. $\tau_{a}=1$ is attained at $A / B=30(\mathrm{eV})^{1 / 2}$.

The cross section for continum photoabsorption by a hydrogenic ion in the non-relativistic 1 imit is (e.g. Bethe and Salpeter 1957)

$$
\sigma_{\text {cont }}^{\text {th }}=\frac{2 \pi^{8}}{3 e^{4}} \alpha \frac{\pi a_{0}^{2}}{z^{2}}
$$

at the threshold energy. In this equation $\alpha \cong \frac{1}{137}$ is the fine structure constant, $a_{0}$ is the radius of the first Bohr orbit, and $z$ is the nuclear charge. Substituting the values of these constants gives $\sigma_{\text {cont }}^{\text {th }}=6.3 \times 10^{-18} \mathrm{~cm}^{2} / \mathrm{z}^{2}$. Thus column densities exceeding $1.0 \times 10^{19} \mathrm{~cm}^{?}, 3.1 \times$ $10^{19} \mathrm{~cm}^{2}, 4.1 \times 10^{19} \mathrm{~cm}^{2}, 1.0 \times 10^{20} \mathrm{~cm}^{2}$ are required for optically thick continuum absorption by the ions 0 VIII, Si XIV, S XVI, and Fe XXVI, respectively. Photons absorped in this way are partially thermalized and reemitted as recombination line radiation. In a recombining qas collisionally 
excited line radiation can be neglected. The recombination line emission equivalent width satisfies the relation $W_{e} \approx \tau^{\tau}$ cont $W_{\max }$ for continuum optical depths $\tau_{\text {cont }} \ll 1$. The maximum equivalent width, attained when $\tau_{\text {cont }} \gg 1$, is defined by

$$
W_{\max }=\varepsilon_{1 \text { ine }} \phi £
$$

where $\varepsilon_{\text {line }}$ is the line energy, $£$ is the fraction of the incident photons which are in the continuum of the emitting ion, and $\phi$ is a number which reflects the efficiency of conversion from photoabsorption to line emission. For our range of spectra $0.1<£<0.4$, and $\phi-0.2$ for case $A$ recombination emission of $L \alpha$ (e.g. Pengelly 1972). Thus, $\tau_{\text {cont }} \geq 0.05$ is required in order to produce $W_{e}>10^{-3} \varepsilon_{1 i n e}$. From figure 2 it is apparent that Fe XXVI is (again) the best candidate for an observable feature. $\tau_{\text {cont }}=0.1$ is exceeded for $A / B>50(e V)^{1 / 2}$. Thus, any recombination emission 1 ine will be weaker than an absorption feature or any scattered emission in a $P$ Cygni profile by a factor $\sim 10$. However, recombination line emission will appear even if the qas flow is predominantly turbulent or random.

We now explore the consequences of possible pressure variations across the two-phase regions. Figure 3 shows the result of evaluating equation (3) using ion fractions and temperatures as a function of $\Xi$ calculated for an $A / B$ $=10(\mathrm{eV})^{1 / 2}$ spectrum. This figure shows that the conclusions reached so far are not qualitatively changed when pressure variations are considered. For $\beta$ $>-2$ the column density is influenced predominantly by conditions at $\Xi=\Xi_{h}$, and increments of $\beta$ result in only small diminutions of the column densities. For example, $N_{F e X X V I}$ never decreases below $10^{17.7}\left(L_{46} P_{14}\right)^{1 / 2} \mathrm{~cm}^{-2}$ for $-2 \leqslant \beta \leqslant+2$, corresponding to a $L \alpha$ analog line optical depth of at least 0.1 using our previous broadening estimate. 


\section{CONSEQUENCES OF TWO PHASE MODELS: COMPTON THICK CASE}

We now consider the case when the ICM is optically thick to Compton scattering. In the context of the two phase models for the broad line region, as outlined in $\S I I$, this can arise if the gas pressure, source luminosity, or source shape is such that the column density through a uniform sphere bounded by $\Xi=\Xi_{h}^{*}$ (c.f. equation (1)) exceeds $1.5 \times 10^{24} \mathrm{~cm}^{-2}$. Our treatment of this problem is influenced by the fact that the gas pressure may be greater closer to the source than in the broad line region. In addition, the two phase models provide only a lower limit for the ICM density--the ICM may be more dense than required by pressure equilibrium (see e.g. Eilek and Caroff 1982). Thus, rather than restricting our choice of ICM properties based on the two phase models, in this section we examine the general effects of Compton scattering by gas near a power law continuum source. In $\S$ IV we discuss a possible scenario whereby this can occur. The effects of Compton scattering on the broad line profiles have been discussed by Shields and McKee (1981); we will ignore them here.

The primary effect of multiple Compton scattering on an AGN power 1 aw is a reduction in the flux at the highest photon energies, relative to the rest of the spectrum. The mean fractional energy shift per scattering of a photon of energy $\varepsilon$ is (e.g. Ross, Weaver, and McCray, 1978).

$$
\stackrel{\Delta \varepsilon}{\stackrel{\Delta \varepsilon}{\rightleftarrows}}=\frac{4 \mathrm{kT} T}{\mathrm{mc} \mathrm{c}^{2}}-\frac{\varepsilon}{\mathrm{mc}} \mathrm{c}^{2}
$$

in the non relativistic limit. For a gas temperature $T \sim 10^{6} \mathrm{~K}$ approximately 150 scatterings are needed to increase the photon energy appreciably, while only -2 scatterings are needed to significantly decrease the enerqy of 250 
keV photons. We will restrict ourselves to optical depths and gas temperatures such that the effects of photon energy gain due to Compton scattering can be neglected.

The consequences of Compton softening for the thermal equilibrium in the broad line region is illustrated in Figure 4 . This figure shows the locus of equilibrium temperatures for a gas illuminated by a spectrum which has passed through a uniform (density) sphere of Compton depths 0,4 , and 8 . The initial spectrum used here is our standard double power 1 aw (Equation 1) with $A / B=10$ $(\mathrm{eV})^{1 / 2}$; the Compton softening was calculated using the procedure described by Illarionov, et al. (1979); the thermal equilibrium was calculated in the same way as in the previous section. For $\tau_{c}=4$, the softening causes the spectrum to have an X-ray to optical ratio resembling that of the softest observed AGN. For $\tau_{c} \geq 4$ the spectrum is softened sufficiently to entirely suppress the thermal instability. Objects with Compton depths in excess of this value may not have broad line clouds, unless an additional heating mechanism exists. Gas near these objects will be distributed uniformly at a temperature of $10^{5}-10^{6} \mathrm{~K}$.

A second consequence of multiple Compton scattering is that the photon path length in the scattering medium will be much greater than the size of the medium. This increases the probability of photoabsorption if the medium is not fully ionized. In the $10^{5} \mathrm{~K}-10^{6} \mathrm{~K}$ gas at the boundary of a Compton thick cloud the photoelectric opacity will be much greater than if the gas were compton thin and the temperature were $10^{7}-10^{8} \mathrm{~K}$. The amplification of this absorption by multiple Compton scatterings can suppress the escaping flux even if the opacity is such that the gas would be optically thin to absorption in the absence of multiple scatterings. Furthermore, the steeply decreasing AGN power law spectrum will preferentially photoionize the low energy 
absorbers $\mathrm{H}$, $\mathrm{He}$, and $\mathrm{C}$. Thus the gas can be transparent between $13.6 \mathrm{eV}$ and the $K$ edge of 0 VIII, near $800 \mathrm{eV}$ (Krolik and Kallman 1984).

A summary description of the effects of simultaneous Comptonization and photoabsorption by ICM gas is as follows; (1) Photons lose a fraction of their energy $\left\langle\frac{\Delta \varepsilon}{\varepsilon}\right\rangle$ at each scattering. The total energy loss on traversing a medium of depth $\tau_{c}$ is approximately $\left.\tau_{c}{ }^{2} \stackrel{\Delta \varepsilon}{\varepsilon}\right\rangle$ (e.g. Illarionov, et al. 1979). (2) The total photon path length in the medium is approximately $\tau_{c} R$, where $R$ is the size of the medium. The optical depth to photoabsorption is therefore $\tau_{a} \simeq \tau_{c}$ $R \kappa_{a}$, where $x_{a}$ is the absorption opacity. (3) The local photon flux in the medium is increased by a factor proportional to $\tau$, owing to the increased path length. In order to model these effects with high accuracy it is necessary to simultaneously solve the equation of radiation transfer, including the effects of energy redistribution due to Compton scattering, together with the ionization and thermal equlibrium equations. Such a procedure is cumbersome and not well suited to probing a wide parameter space le.g. Ross, Weaver, and McCray 1978). We therefore adopt an approximate technique for modeling simultaneous Comptonization and photoabsorption which preserves all of the qualitative behavior of more accurate treatments without necessitating a detailed solution of the full radiative transfer problem. We do so by modifying the program used to calculate the thermal equilibrium results described in $\$ I I$ in the following ways: (1) At each radius step in our spherical clouds a fraction of photons at energy $\varepsilon$ are removed, the fraction being $\underset{\varepsilon}{\stackrel{\Delta \varepsilon}{\rightleftarrows}} \mathrm{d} \tau_{C}^{2}$, where $\mathrm{d} \tau_{C}^{2}$ is the increment of Compton depth squared in that radius step. (2) The local ionizing flux in the interior of the cloud is increased over the Compton thin value by a factor $1+\tau_{c}$, where $\tau_{c}$ is the Compton depth to the cloud boundary. (3) The photoabsorption opacities are increased over the Compton thin values by a factor of $1+\tau_{c}$. As a further 
computational expedient, the clouds are assumed to have constant density rather than pressure. This allows the effect of the cloud boundary to be calculated analytically in the cloud interior, and avoids the need for an iterative solution of the transfer and thermal equilibrium. The appropriate ionization parameter in this case is proportional to the ratio of the radiation flux $F$ at the cloud boundary to the gas density $n$, which we define as $\xi=4 \pi \mathrm{F} / \mathrm{n}$ (Tarter, Tucker, and Salpeter, 1969). The properties of such a Compton thick cloud are determined entirely by the Compton depth $\tau_{c}$, $\xi$, and the shape of the incident continuum.

We have tested our approximate treatment of Comptonization by comparing the spectrum escaping a pure scattering cloud of depth $\tau_{c}=4$ calculated with our technique with that calculated using the formulation of Illarionov et al. (1979). The approximate spectrum is found to be within $20 \%$ of the exact result for photons which have been shifted by more than $\sim 0.5$ Compton wavelengths as they escape. For small wavelength shifts, corresponding to photon energies greater than $\sim 100 \mathrm{KeV}$, our approximate technique significantly underestimates the escaping flux. We attribute this to predominantly non-Gaussian nature of the distribution of energy shifts for one or two scatterings. The error involved is small since even the exact formulation predicts that less than $10 \%$ of the flux emitted above $\sim 100 \mathrm{keV}$ escapes in that energy range from a Comptonizing cloud of $\tau_{c} 22$.

A sample Comptonized and photoabsorbed spectrum is shown in Figure 5 , for $\tau_{c}=10$ and $\log \xi=2.5$. The incident continuum is shown as the dashed curve, and the effects of simultaneous Comptonization and photoabsorption are shown in the solid curves. The effects of photon destruction at the highest energies by Compton scattering and in the 1-10 keV band by photoabsorption are apparent in this figure. Photons below $\sim 800 \mathrm{eV}$ escape the cloud freely for 
this choice of parameters. The energy removed from the $X$-ray band above $\sim 1$ $\mathrm{KeV}$ escapes the scattering and absorbing region at lower energies, either as a result of down-Compton scattering or as reprocessed photo-absorbed radiation (i.e. as soft X-ray and XUV lines and continua from ions such as 0 VIII, C, VI and He II). The detailed dependence of the escaping spectrum on cloud depth and the ionization parameters at the cloud boundary is displayed in Figure 6. In this figure the contours of constant flux in the $13.6 \mathrm{eV}-500 \mathrm{eV}$ energy band (dashed lines) and $500 \mathrm{eV}-4 \mathrm{keV}$ band (solid line) are shown as a function of $\tau_{c}$ and $\xi$. These fluxes are measured relative to their optically thin values, for a spectrum of the form given by equation (1) with $A / B=10$ $(\mathrm{eV})^{1 / 2}$.

For $1 \leqslant \tau_{c} \leqslant 2$ the shape of the escaping spectrum is a sensitive function of $\tau_{c}$, owing to the degredation of the highest energy photons $\left(\varepsilon \sim m c^{2}\right)$ by Comptonization. Since the gas temperature is coupled to the high energy flux via Compton heating, the ionization state and opacity are also sensitive to $\tau_{C}$ in this region. If we define $\xi_{C}$ as the critical ionization parameter below which photoabsorption becomes important, the value of $\xi_{c}$ (inferred from the constant flux surfaces labeled 1.0 in Figure 5 ) is sensitive to $\tau_{c}$, for $\tau_{c} \leqslant 2$. For $2 \leqslant \tau_{c} \leqslant 10$ the effects of Comptonization are complete: further increases of $\tau_{c}$ have negligible effect on the escaping spectrum and on $\xi_{c}$. The effects of preferential ionization of $10 \mathrm{w}$ energy absorbers in this range of $\tau_{c}$ is apparent from the fact that the 0.5 - $4 \mathrm{keV}$ flux decreases to $30 \%$ of its optically thin value for by $\log (\xi) \leqslant 2.2$, while the $13.6 \mathrm{eV}-0.5 \mathrm{keV}$ flux is less strongly affected unless by $\log (\xi) \leqslant 1.8$. The effects of Compton amplification of photoabsorption are apparent for $\tau_{c} \geqslant 10$. In this range of depths the photons in the $0.5-4 \mathrm{keV}$ range can be $90 \%$ absorbed, while less than $50 \%$ of the $13.6 \mathrm{eV}-0.5 \mathrm{keV}$ photons are absorbed. This can 
occur over a range of ionization parameter of roughly a factor of 10 at $\tau_{c}=$ 20 as illustrated by the hatching in Figure 6 . This behavior is consistent with the results of analytic estimates for the transmission coefficient of a Compton scattering, photoabsorbing sphere. Such estimates (see appendix) predict that surfaces of constant transmitted flux will be congruent with $\tau_{c} \alpha \xi$ surfaces, and that photoabsorption will be more important at high photon energies than at lower photon energies.

\section{DISCUSSION}

In addition to accounting for the existence of quasar broad line clouds, the two phase models can be used to predict ICM properties for AGN as a function of continuum luminosity. The cloud filling factor, $f$, is expressed in terms of cloud density, $\mathrm{n}_{\mathrm{C}}$, and total (cloud + ICM) mass $M$ accordina to:

$$
f=\frac{M}{\frac{4 \pi}{3} R^{3} \mu m_{H} n_{C}}
$$

where $\mu$ and $m_{H}$ are the gas mean molecular weight and proton mass, respectively. The broad line region size is given by the ionization parameter

$$
R=\left(\frac{L}{4 \pi C P \equiv}\right)^{1 / 2} \text {, }
$$

so the filling factor is

$$
f=1.7 \times 10^{-3} L_{46}^{-1} M_{4}^{2 / 3} P_{14} \equiv \text {, }
$$

where $L_{46}$ is the source luminosity, $M_{4}$ is the mass in units of $10^{4} M_{0}$ and $P_{14}$ is the pressure in units of $10^{14} \mathrm{~cm}^{-3} \mathrm{~K}$. Thus the filling factor will approach 
unity for $L \sim 10^{43} \mathrm{erg} \mathrm{sec}^{-1}$, in accord with the observed almost total covering of low luminosity Seyfert galaxies (Holt, et al., 1980: Reichert et al. 1984), and the low mean covering factor in OSO's (0smer 1980).

The combined column density of the clouds and ICM is given by $N=n R$, where $\bar{n}$, the mean gas density, is given by (KMT)

$$
\bar{n}=n_{c} f+(1-f) n_{h}
$$

and $n_{c}$ and $n_{h}$ are the cloud and ICM gas densities, respectively. The column density is therefore

$$
N=\frac{3 M}{\mu m_{H}} \frac{P C \equiv}{L}\left(1-\frac{n_{h}}{n_{C}}\right)+\left(\frac{L P}{4 \pi C \equiv}\right)^{1 / 2} \frac{1}{K T_{I C}}
$$

if the pressure is constant across the broad line region. This equation shows that the column density increases proportional to (LP) $)^{1 / 2}$ at hiah luminosity, in accord with equation (4). However, the two phase model also predicts a column density increase proportional to $(L / P)^{-1}$ at low luminosity, if $M$, and $\equiv$ are independent of luminosity. Thus, we expect Compton thick ICM qas both at very high luminosity, and at very low luminosity. This is consistent with the idea that Seyfert 2 nuclei are lower luminosity versions of Seyfert 1's, and that their continuum and broad line properties are dominated by the effects of Comptonization and Compton-amplified photoabsorption. This is also consistent with the apparent cutoff in the quasar luminosity function above $\sim 10^{48} \mathrm{erg}$ $\sec ^{-1}$ (e.g. Kuhr et al. 1983). Very high luminosity quasars are likely to have their emission lines smeared into unobservability by Compton scattering, 
as noted by Shields and Mckee (1981), in addition to having a weakened or absent thermal instability.

The observed properties of Seyfert 2 galaxies include weakened or absent broad emission lines (Weedman 1976), weak or absent X-ray flux in the 0.5-5 $\mathrm{keV}$ (Einstein Observatory) X-ray band (Lawrence and Elvis 1982), and weak or absent X-ray flux at energies greater than $5 \mathrm{keV}$ (Piccinotti et al. 1982). These galaxies also show narrow emission lines which resemble those of higher 7 uminosity Seyfert 1 galaxies. The Comptonized and photoabsorbed spectra presented in $\$$ III have sufficient flux escaping in the $13.6 \mathrm{eV}-800 \mathrm{eV}$ energy range to penetrate a column density $\sim 10^{21} \mathrm{~cm}^{-2}$ in narrow line clouds and produce narrow lines similar to those observed (Halpern and Steiner, 1983).

Other observed properties of Seyfert 2's support the idea that they are optically thick to Compton scattering. Optical polarization studies (Antonucci, 1983) have revealed that the linear polarization of Seyfert 2's is preferentially perpendicular to the radio structure in these objects, while Seyfert l's show polarization which is preferentially parallel. If the qas in these galaxies is distributed in a flattened cloud whose normal is coincident with the radio structures, then the observed polarizations are consistent with optically thick and optically thin Compton scattering for Seyfert 2's and Seyfert 1's, respectively. Al though our discussion in this paper is based on an assumed spherical gas distribution, the qualitative results will be unchanged if the distribution is flattened slightly. As shown by Angel (1969), an axial ratio of $1 / 3$ is adequate to account for the degree of polarization of a few percent observed from Seyfert 2's. Finally, we note that the optical continuum of Seyfert 2 's exhibits variability 1 imits that are less than those of Seyfert 1's. This behavior is consistent with the effects 
of Compton scattering smearing variability on timescales less than the broad 1 ine region light crossing time, $\sim 1$ week.

Our model results show that a Compton depth of -1 is sufficient to suppress the two phase instability, while a depth of 5-10 is required to suppress the escaping $X$-ray flux at $\geq 1 \mathrm{keV}$. Therefore, if the ensemble of known active galaxies has a distribution of Compton depths spanning this range, the range of AGN properties should include those with both broad lines and simple power law X-ray spectra (e.g. quasars and Seyfert 1's), those with neither broad lines or power law X-rays (e.g. Seyfert 2's), and those with weak or absent broad lines but apparently normal $X$-ray spectra. Possible examples of this last class of objects include the intermediate Seyfert's, and the narrow emission line galaxies (Mushotzky, 1982). The observed metamorphosis of some intermediate type Seyfert's into objects with no broad lines may also be consistent with the effects of Comptonizaton. For example, the dynamical time scale at the radius of the broad line region in the Seyfert galaxy NGC 4151 is roughly 1 year. On this timescale an influx of material to the broad line region could increase the compton depth sufficiently to suppress the thermal instability and account for the observed disappearance of the broad lines (U1rich, et al. 1984). It should be noted that the X-ray spectrum and the UV/X-ray flux ratio are not observed to vary stronaly in NGC 4151 (Perola et al. 1984).

The results of this paper can be summarized as follows: In \$I we showed that two phase models predict ICM column densities in excess of $10^{23} \mathrm{~cm}^{-2}$ for a wide range of plausible AGN conditions. This conclusion depends only on the requirement that the observed broad line clouds be confined by ICM thermal qas pressure. Furthermore, the degree of ionization of the ICM is predicted to be sufficient to produce potentially observable X-ray line absorption. For 
example an Fe XXVI L $\alpha$ absorption equivalent width of $40 \mathrm{eV}$ will occur for almost any plausible illuminating spectrum and ICM pressure distribution if the ICM pressure and source luminosity satisfy the condition $\mathrm{L}_{46} \mathrm{P}_{14} \geq 1$. Features of this strength will be observable with future X-ray spectroscopy experiments, such as those planned for the Advance X-ray Astrophysics Facility (AXAF) satellite. The absorption lines will only occur if the ICM gas has a net velocity toward or away from the continuum source. Emission lines will have equivalent widths approximately a factor of 10 smaller than for absorption lines, and will be produced even if the gas flow is turbulent or random. Detection of $X$-ray 1 ines from $A G N$ provides a test for the two phase models ofthe broad line clouds, and also serves as a probe of the broad line gas motions.

In $\$ I I I$ we showed that Compton scattering by the ICM can have two effects which will affect both the broad line and X-ray properties of AGN. At moderate Compton optical depths the spectral softening due to Comptonization can suppress the two phase instability which allows the coexistence of broad line clouds and ICM in quasars and type 1 Seyferts. At larger Compton optical depths $\left(\tau_{c} \geq 10\right)$ scattering increases the photon path length sufficiently to cause absorption of photons in the 1-10 keV energy band. Such a medium will also be transparent to photons with energies less than $1 \mathrm{keV}$ if the ICM mass is comparable to that inferred for quasars. We therefore suggest that this scenario accounts for the absence of $X$-rays and broad lines in Seyfert 2 galaxies. This idea is consistent with the polarization and variability properties of Seyfert 2 's as compared to Seyfert 1's. It also arises naturally if the cloud plus ICM mass and ionization parameter are assumed to be the same for all AGN. 
Acknowl edgements:

We thank R. McCray and R. Ross for helpful discussions pertaining to Compton scattering, and A. Szymkowiak for computing assistance. 


\section{APPENDIX}

Analytic Theory of Photoabsorption with Compton Amplification

The equation of ionization equilibrium for an ion whose ionization is controlled primarily by its own photoabsorption is:

$$
\chi_{0} \frac{L}{4 \pi R^{2}} \int_{\varepsilon_{t h}}^{\infty} \frac{d \varepsilon}{\varepsilon} f_{\varepsilon} e^{-\tau(\varepsilon)} \sigma(\varepsilon)=\chi_{+} n_{e^{\alpha}}(T)
$$

where $x_{0}$ is the fractional abundance of the ion in question, $x_{+}$is the franctional abundance of the next higher ionization stage, $L$ is the total incident luminosity, $R$ is the distance from the source, $f_{\varepsilon}$ is the spectral distribution function $\left(\int_{0}^{\infty} f_{\varepsilon} d_{\varepsilon}=1\right)$, $\sigma$ is the photoionization cross section, $\mathrm{n}_{\mathrm{e}}$ is the electron number density, and $\alpha(T)$ is the recombination rate coefficient. If we assume that: (i) $x_{+} \approx 1, x_{0} \ll 1 ;(i i) T$ = const; (iii)

$$
\begin{aligned}
& \text { (iii) } \int_{\varepsilon_{t h}}^{\infty} \frac{d \varepsilon}{\varepsilon} f_{\varepsilon} e^{-\tau(\varepsilon)} \\
& x_{1} \approx \frac{4 \pi \alpha}{f \sigma \xi} e^{\tau} \\
& \text { or } \quad e^{-\tau} d \tau=\frac{4 \pi \alpha n}{f \xi} d R
\end{aligned}
$$

if $n=$ constant this integrates to

$$
1-e^{-\tau}=\frac{4 \pi \alpha}{\text { ef }} \frac{(\operatorname{Ln})^{1 / 2}}{\xi^{1 / 2}}
$$

The transmission coefficient of a Compton thin cloud of Compton depth $\tau_{c}$ and ionization parameter $\xi$ at the boundary is therefore 


$$
T=e^{-\tau}=1-\frac{4 \pi \alpha}{3 f \sigma_{t h}} \frac{{ }^{\tau} c}{\xi}
$$

where $\sigma_{\text {th }}$ is the Thomson cross section. In the Compton thick case the effective photoabsorption cross section is inreased by a factor $\tau_{c}$, so the transmission coefficient becomes

$$
T=e^{-\tau \tau} c=1-\frac{4 \pi \alpha}{3 f \sigma_{t h}} \frac{\tau_{c}}{\xi}
$$

The important conclusions which can be reached from this discussion are that: (i) Surfaces of constant $T$ are congruent to surfaces of $\tau_{c} \alpha \xi$, i.e. diagonal lines, in the $\tau_{c}, \xi$ plane; ( $i i$ ) ions with smaller specific flux in their ionizing continuum, i.e. ions with higher threshold energies, will be more likely to recombine and cause photoabsorption than will ions subject to larger ionizing fluxes. Thus at a given value of $\tau_{c}$ and $\xi$, ions with high energy photionization threshold (e.g. $\varepsilon>1 \mathrm{keV}$ ) are more likely to cause optically thick photoelectric absorption than will ions with low energy threshold (e.g. $\varepsilon<1 \mathrm{keV}$ ). 


\section{REFERENCES}

Angel, J. R. P. , 1969, Ap.J. 158219.

Antonucci, R. R. J., 1983, Nature 303, 158.

Basko, M. M., Komberg, B. V., and Moskalenko, E. I., 1980, preprint.

Bel tametti, M., 1981, Ap. J., 250, 18.

Ejlek, J. and Caroff, L., 1976, Ap.J. 208, 887.

Elvis, M. and Fabbiano, G. 1984, Ap.J. 280, 91.

Field, G. G., 1965, Ap.J. 142, 431.

G1ass, I., 1982, M.N.R.A.S., 197, 1067.

Glass, I., Moorwood, A., and Eichendorf, W., 1982, Ast. and Ap. 107, 276.

Halpern, J. and Steiner, J., 1983, Ap.J. Lett. 269, 137.

Holt, S. S., Mushotzky, R. F., Becker, R. H., Boldt, E. A., Serlemitsos, P.

J., Szymkowiak, A. E., and White, N. E., 1980, Ap.J. (Letters), 241, L13.

Illarionov, A., Kallman, T. R., McCray, R. A., and Ross, R. R., 1979, Ap.J. 228, 279.

Kallman, T. R., and McCray, R. A., 1982, Ap.J. Supp. 50263.

Kallman, T. R., 1984, Ap.J. 280, 269.

Krolik, J. H., McKee, C. F., and Tarter, C. B., 1982, Ap.J. 249, 422.

Krolik, J. H., and London, R., 1983, Ap. J. 267, 18.

Krolik, J. H., and Kallman, T. R., 1984, Ap.J. in press.

Kuhr, M., Liebert, J. W., Strittmatter, P. A., Schmidt, G. D., and MacKay, C., 1983, Ap. J. Lett. 275, 133.

Kwan, J., and Krolik, J. H., 1981, Ap.J. 250, 478.

Lawrence, A., and Elvis, M., 1982, Ap.J. 256, 410.

Malkan, M., and Sargent, W. L. W., 1982, Ap.J. 254, 22.

Mathews, W. G., 1974, Ap.J. 189, 23. 
Mathews, W. G., 1982, Ap. J. 252, 39.

McCray, R. A., 1979, in Active Galactic Nuclei ed. C. P. Hazard and S. Mitton, Cambridge University Press, Cambridge, U.K.

Mushotzky, R., 1982, Ap.J., 256, 92.

Mushotzky, R. F., Marshall, F. E., Boldt, E. A., Holt, S. S., and Serlemitsos,

P. J., 1980, Ap. J., 235, 377.

Osmer, P., 1980, Ap. J. Supp. 42, 523.

Penge17y, R. M., 1964, M.N.R.A.S. 127, 145.

Perola, C. et al., 1984 preprint.

Piccinotti, G., Mushotzky, R., Boldt, E., Holt, S., Marshall, F. E., Serlemitsos, P., and Shaffer, R. E., 1982, Ap. J., 261, 42.

Primini, F. A., Cooke, B. A., Dobson, C. A., Howe, S. K., Scheepmaker, A., Wheaton, W. A., Lewir, W. H. G., Baity, W. A., Gruber, D. E., Matteson, J., and Peterson, L. E., 1979, Nature, 278, 234.

Reichert, G., Mason, K., Thorstensen, J., and Bowyer, S., 1982, Ap.J. 260, 437.

Reichert, G., 1984, private communication.

Ross, R. R., Weaver, R., and McCray, R., 1978, Ap. J., 219, 292.

Rothchild, R., Mushotzky, R., Baity, W., Gruber, D., Matteson, J., and Peterson, L. B., 1983, Ap.J. 254, 22.

Shields, G., and McKee, C. F., 1981, Ap. J. Lett., 246, L57.

Tarter, C. B., Tucker, W., and Salpeter, E., 1969, Ap. J., 156, 943.

U1rich, M. H., et al., 1984, MNRAS, 206, 221.

Weedman, D., 1976, Ann. Rev. Ast. and Ap., 15, 69.

Weymann, R., 1976, Ap. J., 208, 286.

Worral1, D. M., Mushotzky, R. F., Boldt, E. A., Holt, S. S., and Serlemitsos, P.J., 1979, Ap.J. 232, 683. 
Wu, C. C., Boggess, A., and Gu11, T. R., 1983, Ap.J. 266, 28.

Zamorani, G., et a1., 1981, Ap.J., 245, 357. 


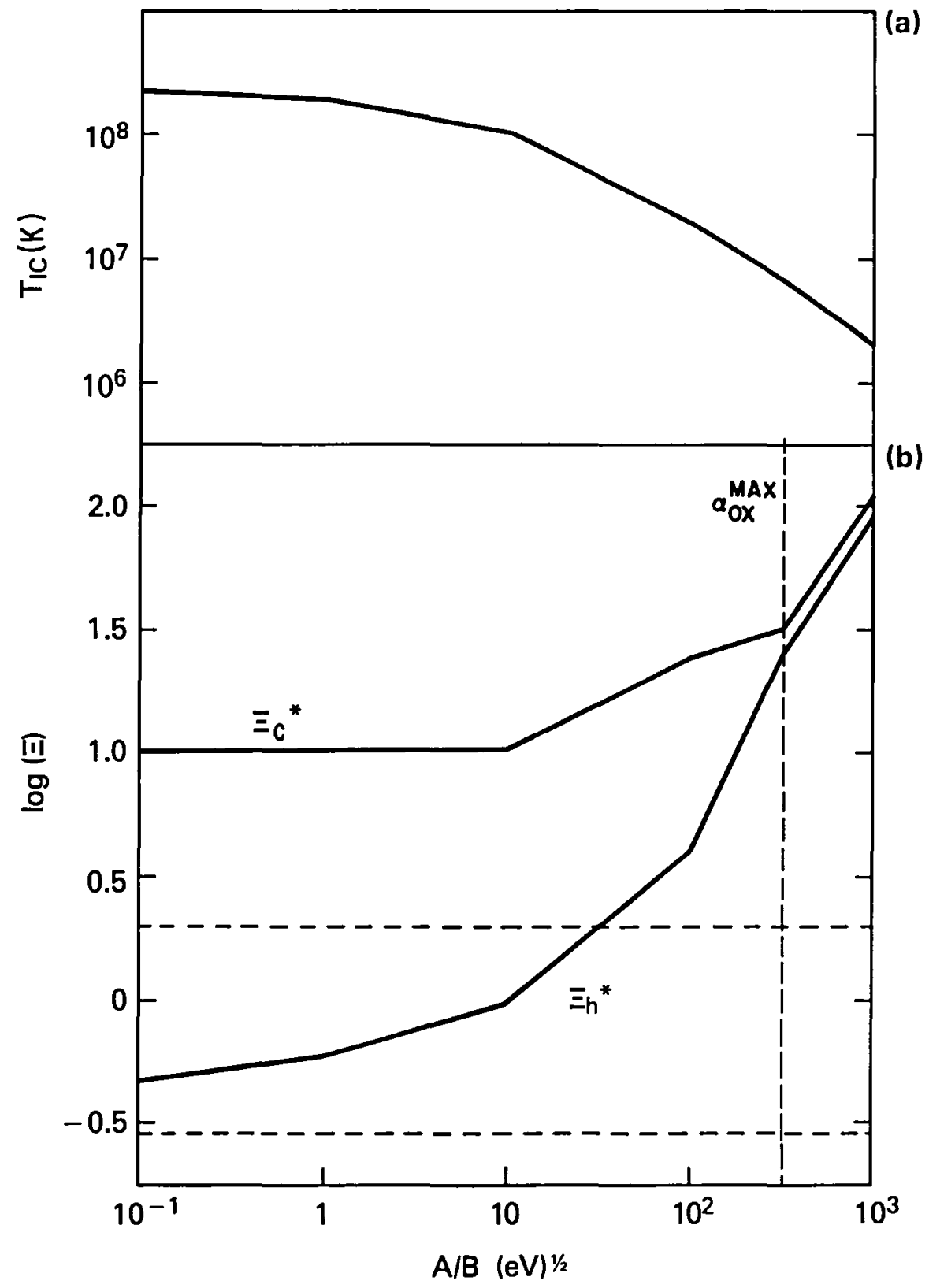

Figure 1(a) Compton Temperature $\left(\mathrm{T}_{\mathrm{IC}}\right)$ as a function of the spectral softness parameter, $\mathrm{A} / \mathrm{B}$ as defined in equation (1)

Figure 1(b) - The limitıng ionization parameters bounding the two phase region, $\Xi_{\mathrm{c}}^{*}$ and $\Xi_{\mathrm{h}}^{*}$, plotted as functions of the spectral softness parameter, A/B Dashed lines denote the allowed region of ionization parameter space as inferred from emission line ratios and photoionization models for the broad line clouds (KMT). The value of A/B which corresponds to the maximum value of $\alpha_{o x}$ is shown as a dashed line 


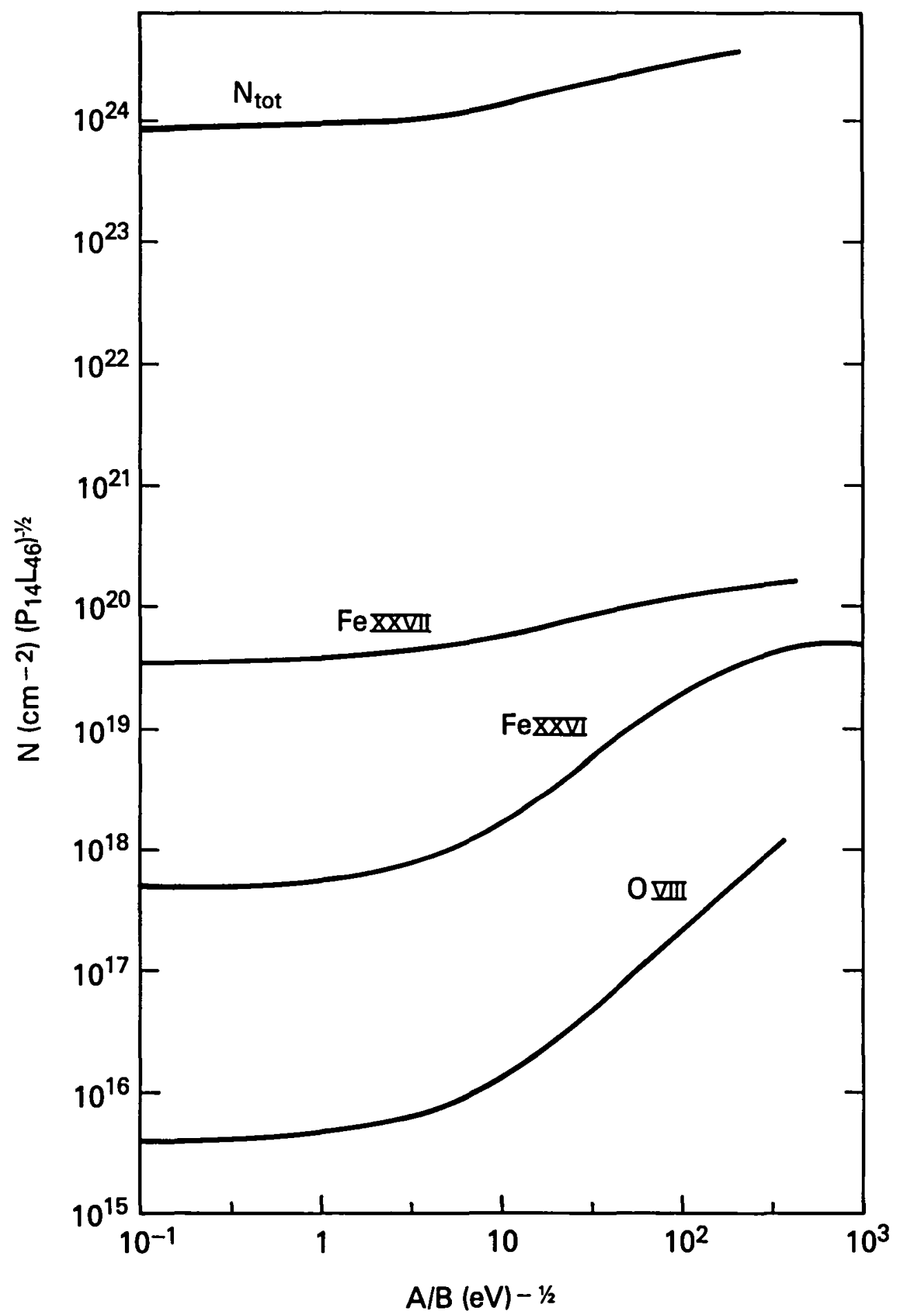

Figure 2. Ion column densities as a function of spectral softness parameter, for the 1ons O VIII, Fe XXVI, and Fe XXVII The total nucleon column density is also shown 


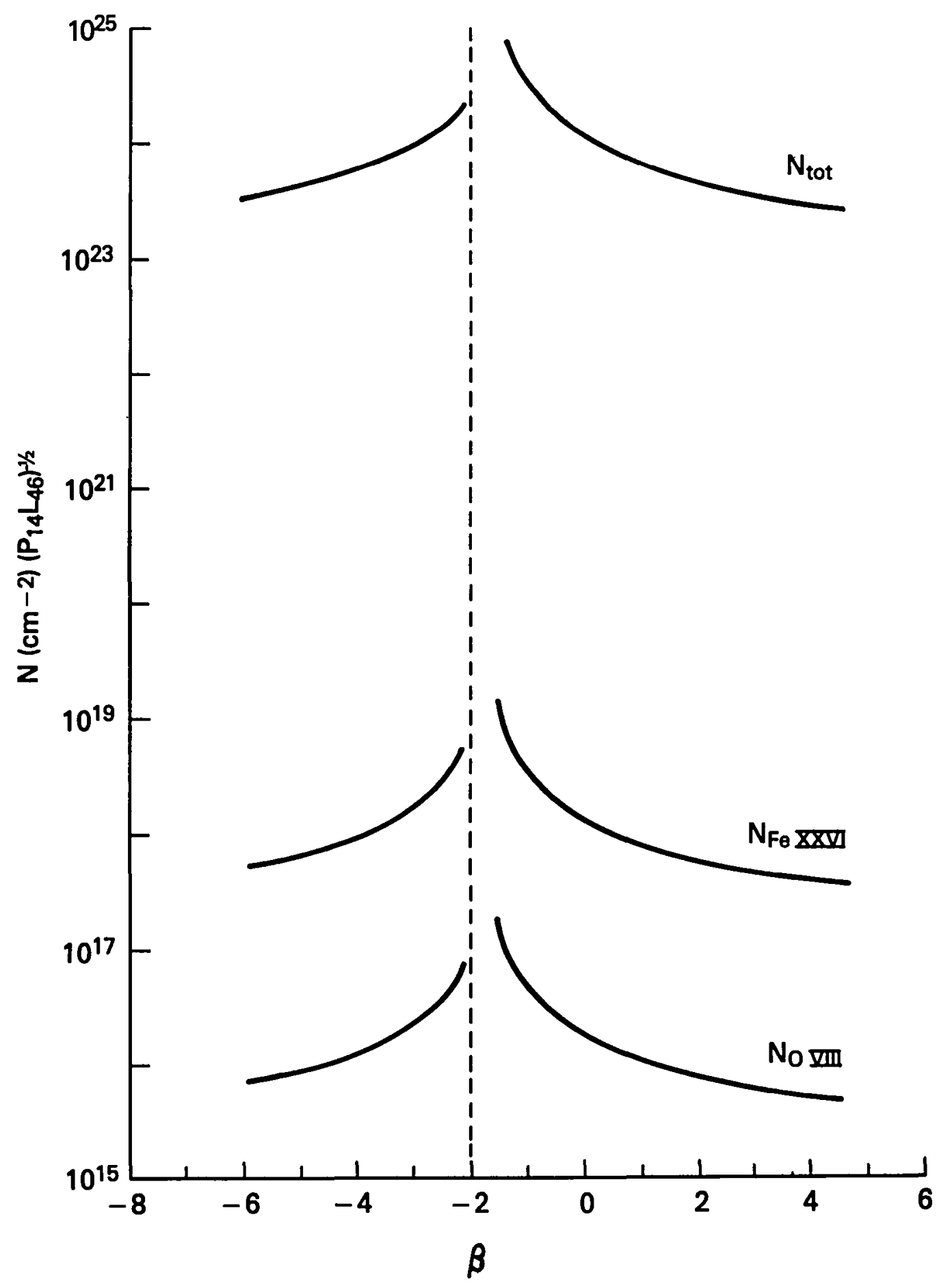

Figure 3. Ion column densities as a function of $\alpha$, the logarithmic pressure gradient in the broad line region. 


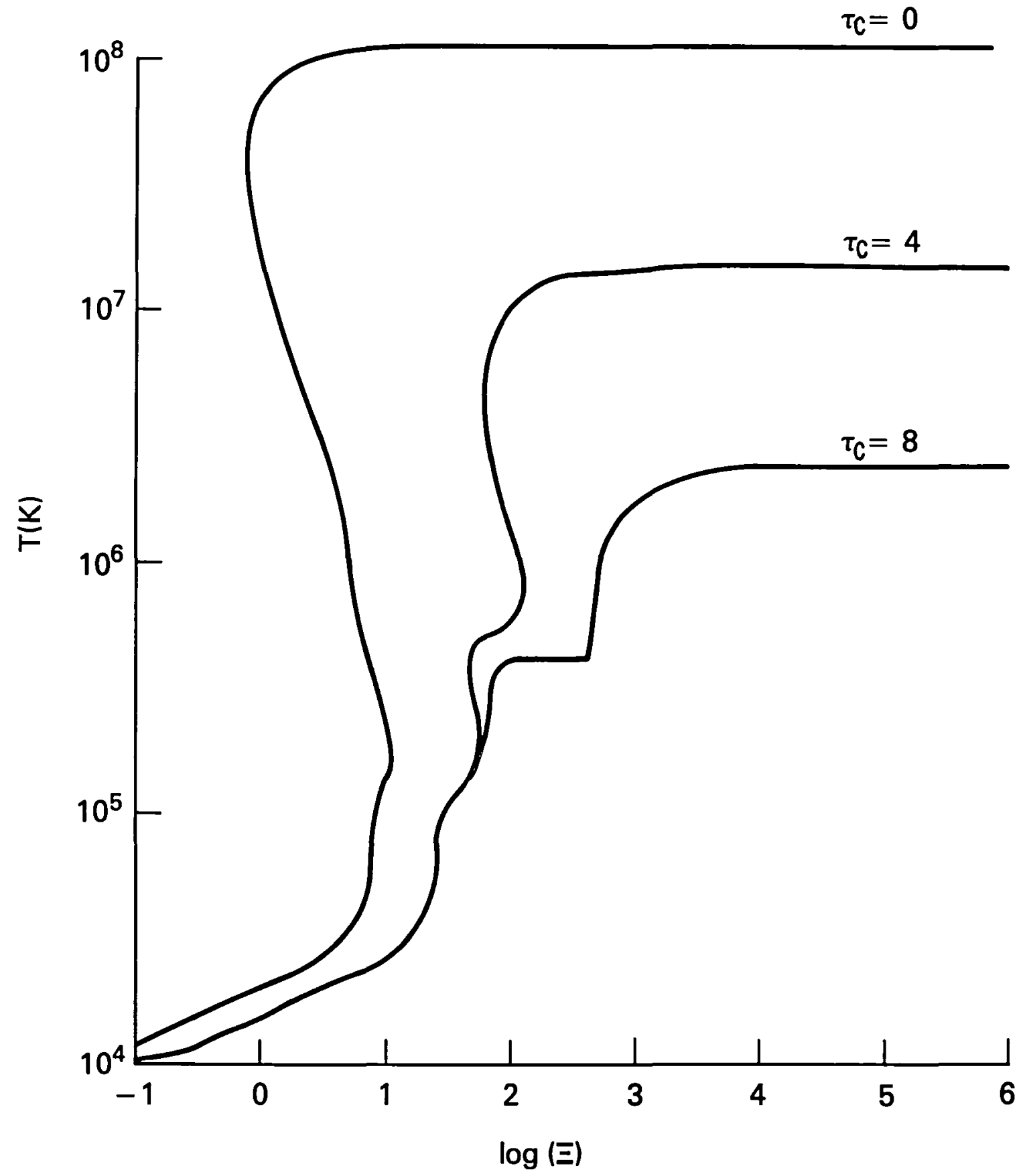

Figure 4 Gas temperature as a function of ionization parameter for an optically thin gas llluminated by a spectrum defined by equation (1) with $A / B=10(\mathrm{ev})^{1 / 2}$ which has passed through a pure scattering cloud of Thomson depth 0,4 and 8 . 


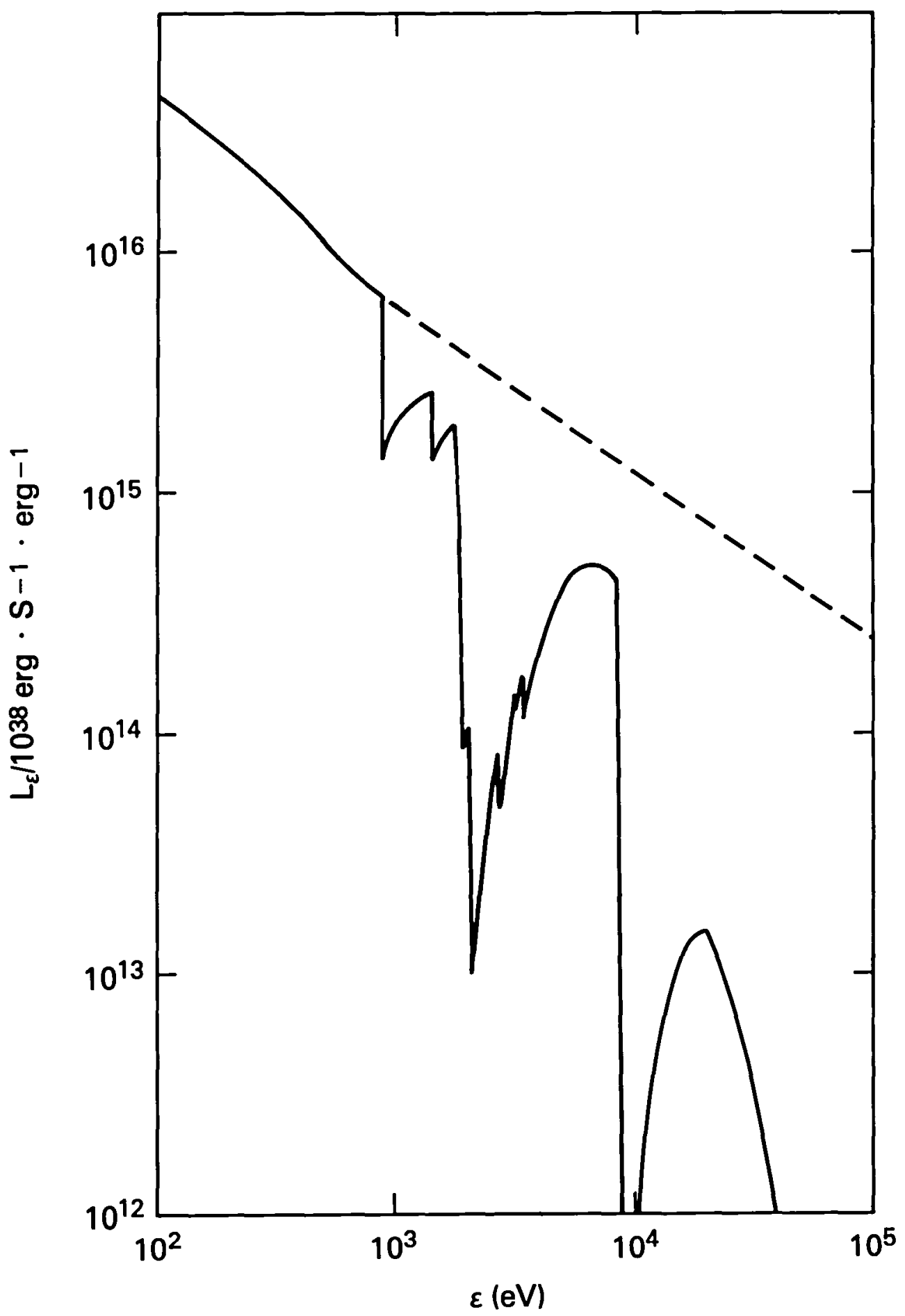

Figure 5. Spectrum escaping a Compton scattering and photoabsorbing cloud with Thomson depth $\tau_{\mathrm{c}}=10$, and ionization parameter $\log \zeta=25$ at the outer boundary. The incident spectrum is of the form of equation (1) with $A / B=10(\mathrm{eV})_{1 / 2}$ 


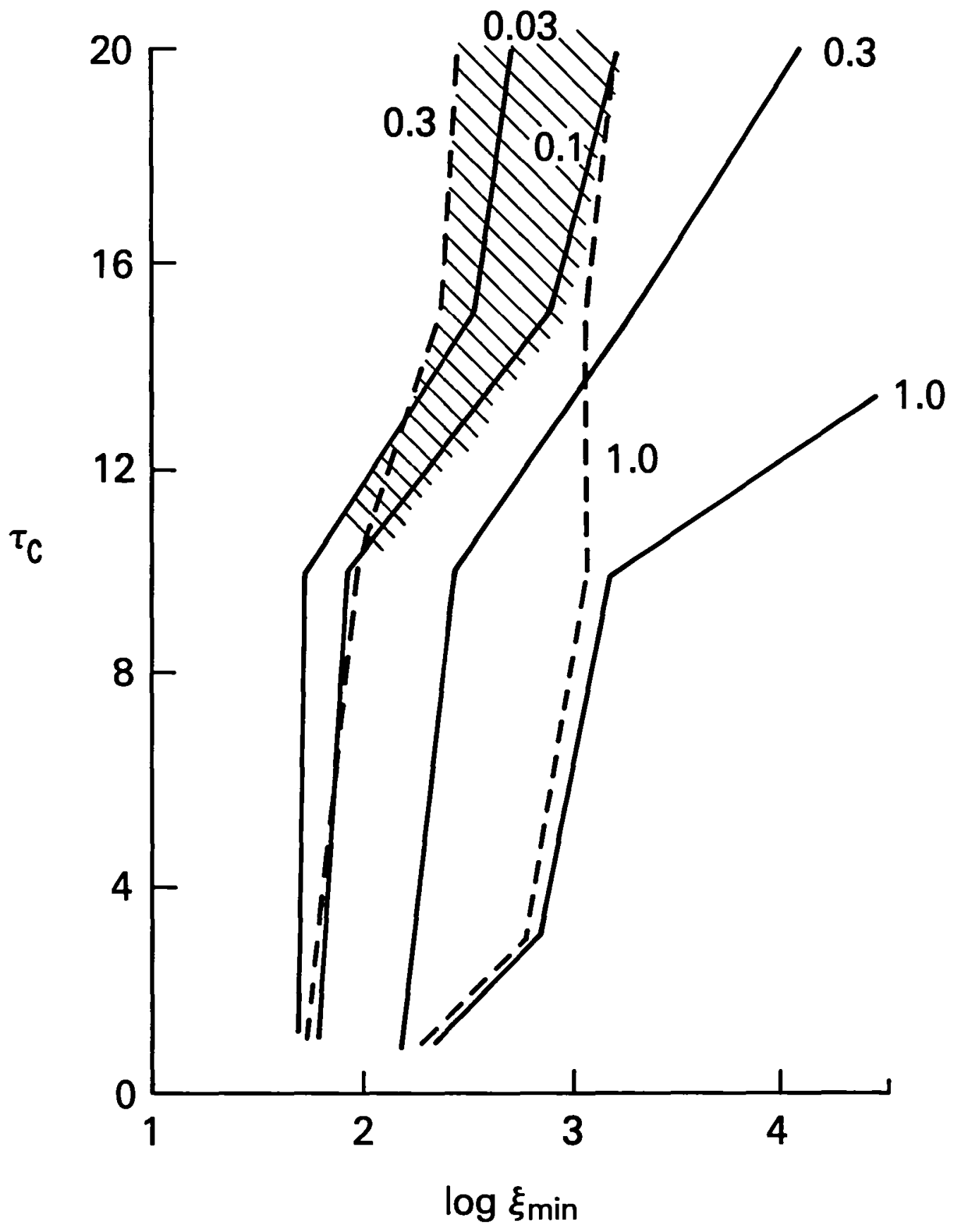

Figure 6. Contours of constant flux in the $136 \mathrm{eV}-05 \mathrm{keV}$ (dashed) and $05 \mathrm{keV}-4 \mathrm{keV}$ (solid) energy bands as a function of cloud Thomson depth, $\tau_{\mathrm{c}}$, and boundary ionization parameter, $\zeta$. Curves are labeled with the fluxes relative to the incident spectrum, which is assumed to be the same as in Figure 5 In the hatched region the emergent spectrum will resemble that shown in Figure 5 
Authors' Addresses

T. Kallman, Code 665

R. Mushotzky, Code 661

Laboratory for High Energy Astrophysics

NASA/Goddard Space Flight Center

Greenbelt, MD 20771 


\begin{tabular}{|c|c|c|c|c|}
\hline $\begin{array}{l}1 \text { Report No } \\
\text { NASA TM-86176 }\end{array}$ & \multicolumn{2}{|c|}{2 Government Accession No } & \multicolumn{2}{|c|}{3 Recipient's Catalog No } \\
\hline \multirow{2}{*}{\multicolumn{3}{|c|}{$\begin{array}{l}4 \text { Title and Subtitle } \\
\text { Consequences }\end{array}$}} & \multicolumn{2}{|c|}{\begin{tabular}{|l}
5 Report Date \\
March 1985
\end{tabular}} \\
\hline & & & \multicolumn{2}{|c|}{ 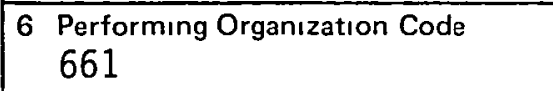 } \\
\hline \multicolumn{3}{|c|}{$\begin{array}{l}7 \text { Author(s) } \\
\text { T. Kallman and R. Mushotzky }\end{array}$} & \multicolumn{2}{|c|}{$\begin{array}{l}\text { 8. Performing Organızation Report No } \\
85 \mathrm{~B} 0237\end{array}$} \\
\hline \multirow{3}{*}{\multicolumn{3}{|c|}{$\begin{array}{l}\text { 9. Performıng Organızation Name and Address } \\
\text { Laboratory for High Energy Astrophysics } \\
\text { NASA/Goddard Space Flight Center } \\
\text { Greenbelt, MD } 20771\end{array}$}} & \multicolumn{2}{|c|}{10 Work Unıt No } \\
\hline & & & \multicolumn{2}{|c|}{11 Contract or Grant No } \\
\hline & & & \multicolumn{2}{|c|}{13 Type of Report and Perıod Covered } \\
\hline \multicolumn{3}{|c|}{ 12. Sponsorıng Agency Name and Address } & \multirow{2}{*}{\multicolumn{2}{|c|}{ Technical Memorandum }} \\
\hline \multirow{2}{*}{\multicolumn{3}{|c|}{$\begin{array}{l}\text { National Aeronautics and Sp } \\
\text { Administration } \\
\text { Washington, D.C. } 20546\end{array}$}} & & \\
\hline & & & \multicolumn{2}{|c|}{14 Sponsorıng Agency Code } \\
\hline \multicolumn{5}{|c|}{$\begin{array}{l}15 \text { Supplementary Notes } \\
\text { Accepted for publication in The Astrophysical Journal }\end{array}$} \\
\hline \multicolumn{5}{|c|}{$\begin{array}{l}16 \text { Abstract } \\
\text { We discuss models for hot gas in the broad line region of active } \\
\text { galactic nuclei. The results of the two phase equilibrium models for con- } \\
\text { finement of broad line clouds by Compton heated gas as described by Krolik, } \\
\text { Mckee and Tarter (1982) are used to show that high luminosity quasars are } \\
\text { expected to show Fe XXVI La line absorption which will be observable with } \\
\text { spectrometers such as those planned for the future X-ray spectroscopy experi- } \\
\text { ments. Two phase equilibrlum models also predict that the gas in the broad } \\
1 \text { ine clouds and the confining medium may be Compton thick. We show that } \\
\text { the combined effects of Comptonization and photoabsorption can suppress both } \\
\text { the broad emission lines and X-rays in the Einstein and HEAO-1 energy bands. } \\
\text { The observed properties of such Compton thick active galaxies are expected } \\
\text { to be similar to those of Seyfert } 2 \text { nuclei. The implications for } \\
\text { polarization and variability are also discussed. }\end{array}$} \\
\hline \multicolumn{2}{|c|}{$\begin{array}{l}17 \text { Key Words (Selected by Author(s)) } \\
\text { galaxies: nuclei--quasars--radiation } \\
\text { mechanisms }\end{array}$} & \multicolumn{3}{|c|}{$\begin{aligned} 18 \text { Distribution Statement } & \\
\text { Unclassified }- & \text { Unlimited } \\
& \text { Subject Category } 90\end{aligned}$} \\
\hline $\begin{array}{l}19 \text { Security Classif. (of this report) } \\
\text { Unclass ified }\end{array}$ & $\begin{array}{r}20 \text { Security Cla } \\
\text { Uncl }\end{array}$ & $\begin{array}{l}\text { sif (of this page) } \\
\text { ssified }\end{array}$ & $\begin{array}{c}21 \text { No. of Pages } \\
40\end{array}$ & $\begin{array}{r}22 \begin{aligned} \text { Price }^{*} \\
\mathrm{A03}\end{aligned} \\
\end{array}$ \\
\hline
\end{tabular}

*For sale by the National Technical Information Service, Springfield, Virginia 22161 
End of Document 